\title{
Focused spatial attention is independent of rapid resumption of an interrupted search
}

\author{
Alejandro Lleras \\ University of Illinois at Urbana-Champaign, Urbana, Illinois \\ AND \\ JAMES T. ENNS \\ University of British Columbia, Vancouver, British Columbia, Canada
}

\begin{abstract}
In three experiments, we examined possible relationships between the spatial focus of attention and the rapid resumption of a visual search following a brief interruption. In Experiment 1, we tested the role of involuntary (exogenous) spatial orienting to one region (quadrant) of a search display; in Experiment 2, we tested the role of voluntary (endogenous) spatial orienting to the same region; and in Experiment 3, we tested the role of voluntary orienting to the specific location in which the target item appeared. All three experiments indicated that spatial orienting speeds correct responding and greatly increases the probability of search success in the look immediately following the presentation of a spatial cue. However, these benefits of spatial cues were also shown to be completely independent of the rapid resumption effect, which depends on observers' forming a perceptual hypothesis about a target in one look, but being unable to confirm that hypothesis until a second one (Lleras, Rensink, \& Enns, 2005).
\end{abstract}

A brief glance at a scene can result in a rich visual experience, one that does not end when the scene vanishes from view. Following even a brief glimpse, humans are able to report many details if probed within $100 \mathrm{msec}$ (Sperling, 1960), and they are able to detect changes made to a scene after $200 \mathrm{msec}$ if the changes involve objects that were the focus of attention during the initial glimpse (O'Regan, Deubel, Clark, \& Rensink, 2000). Here we examine the relations between focused spatial attention and the processing that occurs following a brief glimpse at a scene.

In addition to documenting the rich and structured memorial content of the visual information in a brief glance (see, e.g., Henderson \& Hollingworth, 2003), recent studies have focused on an important predictive aspect of the processing that occurs following a brief glimpse. This is highlighted in the phenomenon of rapid resumption, which is a pattern of responding that occurs when observers perform a visual search task in which brief glances at a scene (e.g., a 100-msec look) are interrupted by blank screens (e.g., a 900-msec wait). Observers are generally quite good at completing interrupted searches of this kind, being able to identify the target within three or four glances at the display and with accuracy levels of $95 \%$ or more (Lleras, Rensink, \& Enns, 2005). The predictive aspect of their processing is revealed in the high proportion of extremely short identification latencies that occur following re-presentation of the display.
Detailed analyses reveal two distinct phases in the response time (RT) distributions that occur following interrupted looks. There is an early phase of correct responding that begins around $100 \mathrm{msec}$ and peaks at 200-299 msec. Then there is a later phase of responding that peaks in the range of 500-699 msec. Illustrations of these two phases can be seen in the middle panels of the figures that present the RT distributions from each experiment in the present study (Figures 3, 5, and 7). In contrast to the pattern of responses that occur following a single glance at a scene ( $<2 \%$ of responses from 0 to $499 \mathrm{msec} ;>98 \%$ of responses from 500 to $999 \mathrm{msec}$; see top panels of Figures 3, 5, and 7), it is common for subsequent glances to result in $30 \%-50 \%$ of responses occurring in the early phase $(0-499 \mathrm{msec})$. This is the operational definition of rapid resumption used in the present study.

We interpret rapid resumption as evidence for the existence of implicit perceptual predictions made in the context of a reentrant processing theory of visual awareness of Di Lollo, Enns, and Rensink (2000). According to this theory, perception is an iterative process whereby information is processed at several levels, most notably a higher level associated with object representations and a lower level associated with precategorical sensory input. Perceptual awareness is achieved once a prediction about a candidate object is created and confirmed against the current sensory input. Notably, a precondition for awareness is that the reentrant activity in the system matches the

J.T. Enns, jenns@psych.ubc.ca 
sensory input. If there is no match, perceptual awareness of the object generating the prediction does not occur, and the system generates a new prediction regarding the current sensory information.

When this theory is applied to visual search (Enns \& Lleras, 2008), it is our view that, after only a few trials in a search task (e.g., looking for a $\mathrm{T}$ among Ls), observers form detailed representations of the target in its various orientations and colors. Yet despite holding these memorial representations, a first glimpse of the display rarely elicits a response in less than $500 \mathrm{msec}$. It takes this long because, even when the observer's eye registers the target, it is still necessary to activate and test a hypothesis about it, in order both to distinguish an actual target from its memorial representation (i.e., to see rather than to merely imagine it) and to prepare the appropriate motor response. Rapid resumption responses following an interrupted look are able to escape some of these time-consuming steps because the information in the first look has been sufficient to activate a hypothesis and to predict what will be seen next, even though the step of confirming the hypothesis must await the reappearance of the display. The central idea is that merely having to confirm a prediction when the display reappears takes less time than activating a perceptual prediction and then confirming it.

The hypothesis that rapid resumption is an index of prediction has been tested in several ways. (1) Rapid resumption does not occur if the scene is not re-presented following a single look (Lleras et al., 2005, Experiment 5), suggesting that the phenomenon occurs when a prediction is confirmed, not merely when a correct hypothesis is entertained. (2) Rapid resumption fails to occur when the first look is a view of all the distractor items in a display (minus the target) and the second look includes the target (Lleras, Rensink, \& Enns, 2007, Experiment 1). This finding is consistent with observers' making a prediction about a particular object rather than about the task context or the scene as a whole. (3) Rapid resumption does not occur when the location of the target is relocated randomly from look to look, consistent with a prediction's being made about an object that is expected in a given location (Lleras et al., 2005, Experiment 6). (4) Rapid resumption still occurs when incidental features of the target item are changed from look to look, but it fails to occur when the changed features are relevant to the identification response that must be made to the target (Lleras et al., 2007, Experiments 3 and 4). This is consistent with the prediction's being made quite specifically about responserelevant features of the target. (5) Placing the target object at the observer's point of fixation in the scene guarantees a high rate of search success following a single look, but it does not produce fast responses to the target on the first look. Rapid resumption in gaze-contingent experiments occurs only on the second look at the target, following a first look that is unsuccessful (van Zoest, Lleras, Kingstone, \& Enns, 2007).

Although our initial discovery of rapid resumption was serendipitous (Lleras et al., 2005), we have since found that the early-phase responses are a ubiquitous feature of visual search tasks (1) that have some probability of not being completed in a single glance at a display and (2) for which the observer has a reasonable expectation that the display will reappear following an interruption. So far, this has been shown in searches for Ts among Ls (as in the present study), search for faces of strangers, friends, and oneself (Riebe \& Enns, 2008), searches that involve targets on every trial that must be discriminated from one another (as in the present study), and searches that involve both target-present and target-absent trials (Riebe \& Enns, 2008). It also seems that we have had an easier time finding variations on the basic interrupted search task that preserve the rapid resumption phenomenon (e.g., randomly varying the duration of the interruption, relocating all the distractors from look to look) than we have had in attempting to find conditions that eliminate the effect. To date these conditions have included randomly relocating only the target from look to look (Lleras et al., 2005), changing a response-relevant feature of the target from look to look (Lleras et al., 2007), and mixing a large number of singlelook trials among multiple-look trials (Lleras, Rensink, \& Enns, 2004).

The present study examines the relationship between focused spatial attention and rapid resumption. Investigating this relationship is important, because it is well established that, when spatial attention is prepared in advance of a visual stimulus, perception becomes both faster and more accurate (Luck \& Vecera, 2002; Yeshurun \& Carrasco, 1999), the effects of backward masking are sharply reduced (Enns, 2004), and success in change detection experiments is vastly improved (e.g., O'Regan et al., 2000; Rensink, 2000). It is therefore worth studying the relationship between rapid resumption and spatial attention, if only because of these established empirical relationships. They suggest that a major contributing factor to the fast responses following an interrupted look may be that attention is already focused on a particular spatial location when the target reappears. However, there is a deeper theoretical reason that makes this relationship important to study too. Lleras et al. proposed that rapid resumption responses are different in kind from the responses that occur after $500 \mathrm{msec}$; they are not merely responses that have been speeded by the appropriate prefocusing on a specific spatial location. If this is so, manipulations of spatial attention should have effects in an interrupted search task that are dissociable from the influences associated specifically with rapid resumption.

We begin this study by considering the role of involuntary (also called exogenous) spatial orienting of attention prior to the onset of one of the looks in an interrupted search task (Experiment 1). It is possible that in the normal course of searching for a target, attention becomes focused on various locations in the scene prior to a look. If the target is then presented near the current focus of attention, it may be sufficient to produce the rapid resumption pattern of responding. However, it is also possible that rapid resumption is associated with voluntary (also called endogenous) orienting to a region of the scene prior to a look. If intentional orienting is required, then rapid resumption may occur when there is an intention to orient to the general region of the display containing the target 
(Experiment 2) or to the specific location in which the target item is presented (Experiment 3). Thus, the main hypothesis that we derive from the possibility that focused spatial attention contributes to rapid resumption is that conditions involving a valid spatial cue in advance of the search display should produce a greater number of earlyphase responses than do conditions involving no cue or an invalid cue. The alternative hypothesis, the one favored by our account of rapid resumption as an index of implicit perceptual prediction (Enns \& Lleras, 2008), is that spatial cues do not increase the likelihood of early-phase responses. To anticipate the results, we report that, although spatial orienting speeds responding and greatly increases the probability of success in the next look, these effects are completely independent of the rapid resumption effect, consistent with reentrant processing theory (Lleras et al., 2005).

\section{EXPERIMENT 1 Nonpredictive Spatial Orienting to the Target Region}

We began by examining the effects of a nonpredictive spatial cue, in order to test for a relationship between involuntary (exogenous) spatial orienting and rapid resumption. In this and the subsequent experiments, observers searched for a single $\mathrm{T}$ among $15 \mathrm{Ls}$ on every trial, until they indicated the color of the $\mathrm{T}$ (blue, red) with a speeded keypress. The search display was shown for 100 -msec looks, interrupted by $900-\mathrm{msec}$ waits, until a response was made. Four small dots presented $400 \mathrm{msec}$ in advance of a look cued observers to attend to one of the four quadrants of the search display. No cue was presented on $25 \%$ of the trials (no-cue baseline), and when the cue was presented on the remaining trials, it appeared randomly prior to the first, second, or third look at the search display. In Experiment 1, the cue was not predictive of the location of the target, allowing us to measure whether there were any differences in rapid resumption when attention was oriented correctly (valid cue) on $25 \%$ of the cued trials versus when attention was misdirected (invalid cue) on $75 \%$ of the cued trials.

\section{Method}

Observers. Sixteen students from the University of Illinois at Urbana-Champaign took part in this experiment in exchange for course credit. All were naive as to the purpose of the experiment and had normal or corrected-to-normal visual acuity. Data from 1 student were disregarded because search accuracy was less than $65 \%$ correct.

Displays and Apparatus. A schematic of the display sequence is shown in Figure 1. All the experiments were generated using MATLAB and the Psychophysics Toolbox (Brainard, 1997; Pelli, 1997). The experiments were run on 3.4-GHz Pentium IV PCs and were viewed on 17-in. CRT monitors. The programs recorded observers' keypresses and RTs. The displays consisted of colored items ( $1 \mathrm{~T}$ and $15 \mathrm{Ls}$ ), which were either red or blue (presented as RGB color values [ $\left[\begin{array}{lll}255 & 0 & 0\end{array}\right]$ and [ $\left[\begin{array}{lll}0 & 0 & 255\end{array}\right]$, respectively) and were displayed on a white background. The items subtended $0.71^{\circ} \times 0.71^{\circ}$ at a viewing distance of $57 \mathrm{~cm}$ and were presented in one of four possible orientations (rotated $0^{\circ}, 90^{\circ}, 180^{\circ}$, or $270^{\circ}$ ), with an equal number of red and blue items on every display. The colored items were assigned randomly to locations within an invisible grid, which was subdivided into four $3 \times 3$ regions (a total of 36 possible locations). Each cell in a grid subtended $1.07^{\circ} \times 1.07^{\circ}$. There was one grid on each quadrant of the display. Each grid occupied an area of $7.5^{\circ} \times 7.5^{\circ}$, and the distance between each grid was $5.36^{\circ}$. The total search array occupied $20.36^{\circ} \times 20.36^{\circ}$ of visual angle, with each item randomly jittered within each grid location. Finally, the four dots used to cue a quadrant of items were black, and they subtended about $0.15^{\circ} \times 0.15^{\circ}$. They were located in the outermost corners of the cued quadrant, without overlapping any items in the display.

Design and Procedure. There were two conditions: uncued ( $25 \%$ of trials) and cued ( $75 \%$ of trials). Uncued trials began with the presentation of the search display for $100 \mathrm{msec}$, which alternated with a blank display of $900 \mathrm{msec}$, until a response was recorded or $16 \mathrm{sec}$ had elapsed. We use the term epoch (Rensink, 2000) to refer to one search display + blank display cycle. Here, all epochs lasted $1,000 \mathrm{msec}$. Cued trials alternated between search and blank displays in the same way, except for the inclusion of the cue dots, which could appear once, before the first presentation of the display $(25 \%$ of trials), the second presentation ( $25 \%$ of trials), or the third presentation ( $25 \%$ of trials). The cue dots were presented for $400 \mathrm{msec}$ immediately preceding a search display. On trials in which the cue display appeared after the start of the first search display, the duration of the blank display was reduced by $400 \mathrm{msec}$, so that the total duration of the epoch continued to be $1,000 \mathrm{msec}$. Finally, the cued

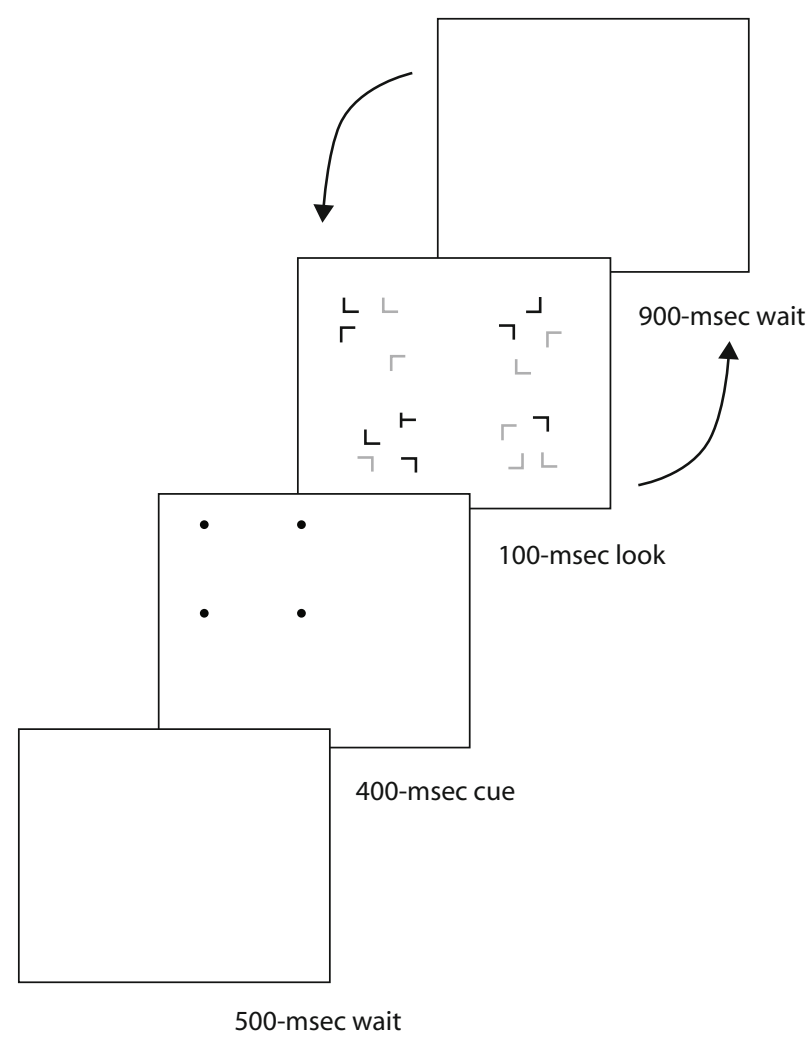

Figure 1. Schematic illustration of the sequence of events in Experiments 1 and 2. Brief glimpses (100-msec looks) of a search display were interrupted by longer blank screens (900-msec waits) until the target (a blue T-shape, in this example) was identified. Randomly selected looks were preceded by a cue (the four dots shown for $\mathbf{4 0 0} \mathbf{m s e c}$ in advance of the search display) to focus attention on one of the four quadrants in which search items appeared. In the example shown, the cue indicates the upper left quadrant but the target is in the lower left quadrant, illustrating an invalidly cued search trial. 


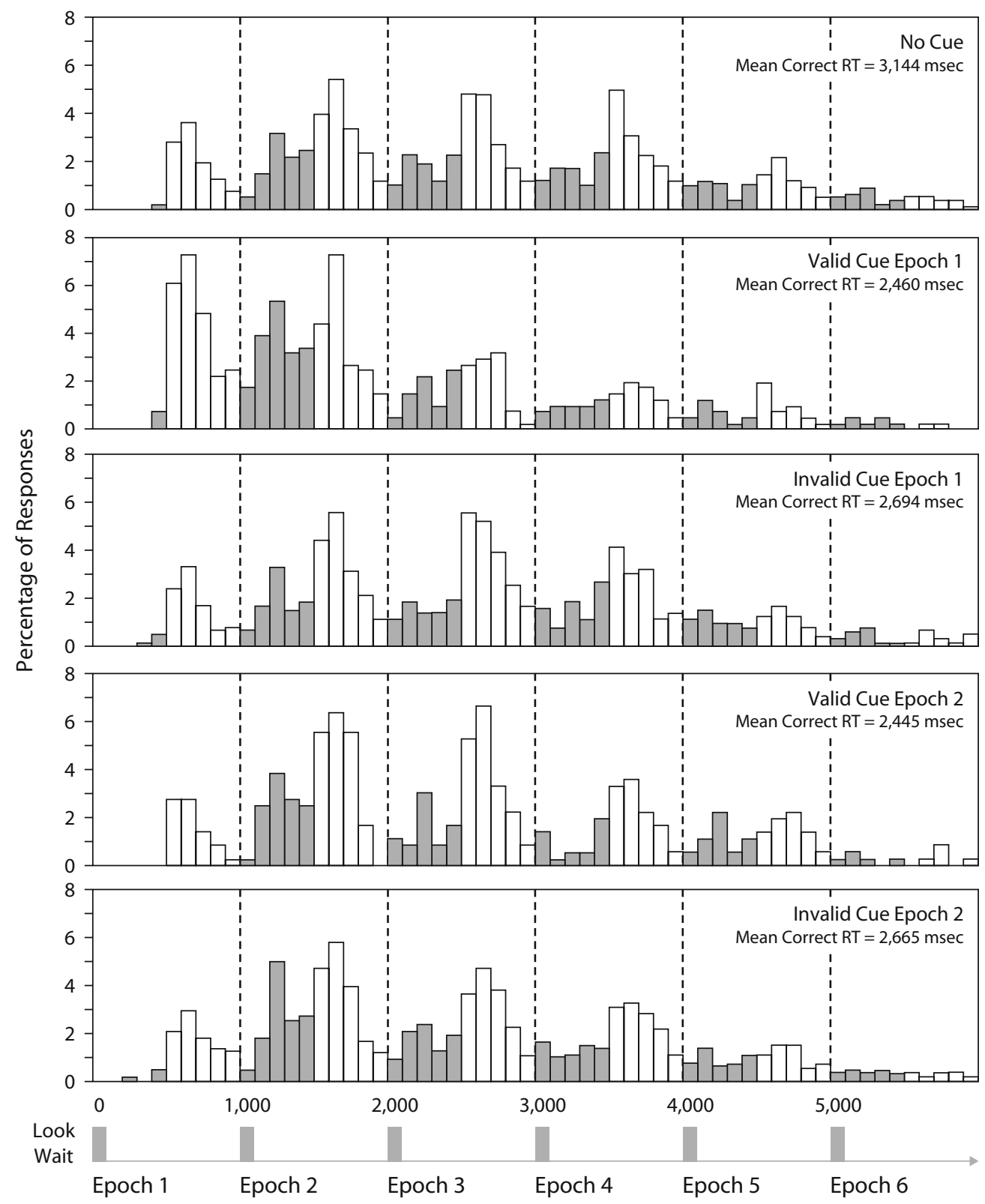

Figure 2. Frequency distributions of correct response times (RTs) in Experiment 1 as a function of various cue conditions. Histograms shaded in gray are responses made within $500 \mathrm{msec}$ of a look, the operational definition of rapid resumption in this study.

quadrant was chosen randomly, and the cue was entirely nonpredictive of the target's region, its specific location, or color. There was an intertrial interval of $1.5 \mathrm{sec}$ between trials. Observers completed one practice set of 10 trials before being tested on 560 trials (eight sets of 64 trials, with short breaks between sets). The experiment took about $45 \mathrm{~min}$ to complete.

\section{Results}

Figure 2 shows the frequency of correct responses for six epochs (epoch = a 100-msec look followed by a 900 msec blank). Five cuing conditions are shown (no cue, valid cue, and invalid cue on Looks 1 and 2). Responses not shown are the few that were made after $6 \sec (<2.4 \%)$ and those made when the cue appeared before Look 3 , because the data for these are so similar to the data derived when the cue preceded Look 2. Accuracy of responses exceeded $94 \%$ overall.

The RT distribution for no-cue trials (Figure 2, top panel) replicated that in previous studies of rapid resumption. Following the first look at the search display (Epoch 1), fewer than $2.4 \%$ of all responses occurred in the first 500msec period (4/230). In contrast, the first 500-msec period following each subsequent look contained more than $35 \%$ of the responses (Look $2=161 / 425$, Look $3=141 / 387$, Look $4=131 / 347$, Look $5=77 / 176$, Look $6=41 / 73$ ). This result establishes that the trials in this experiment on 
which no cue was presented led to the rapid resumption effect found in previous studies with no cue (Lleras et al., 2005, 2007).

The influence of the cue was examined in two ways: (1) its effectiveness in increasing overall search efficiency, measured by mean correct RT, and (2) the cues' influence on the proportion of responses made early in an epoch (rapid resumption) relative to the proportion of responses made later within an epoch.

We estimated overall search efficiency by computing mean correct RT in the various conditions for each observer. An ANOVA comparing the seven cue conditions (no cue, Valid Look 1, Invalid Look 1, Valid Look 2, Invalid Look 2, Valid Look 3, Invalid Look 3) indicated that the mere presence of a cue reduced RT significantly over the no-cue condition (mean RT $=2,588 \mathrm{msec}$ vs. 3,144 msec, respectively) $\left[F(1,14)=9.30, M S_{\mathrm{e}}=\right.$ $249,941, p<.01]$ and that RT was significantly less on valid cue trials than on invalid cue trials (mean RT $=$ $2,489 \mathrm{msec}$ vs. $2,687 \mathrm{msec}$, respectively) $[F(1,14)=4.58$, $\left.M S_{\mathrm{e}}=192,736, p<.05\right]$. These analyses confirmed that the nonpredictive spatial cue generally decreased search times, as in many previous studies.

The relative distribution of correct responses within an epoch is shown in Figure 3 for the various cuing conditions. To facilitate comparison among epochs differing greatly in overall frequency of response, all bin frequencies are plotted relative to those in the bin with the highest peak (in all three panels, this is Bin 7, or 600-699 msec), which has been set to 1.0. The first panel shows that the relative RT distributions in the first epoch contain very few early-phase responses (RT $<500 \mathrm{msec}$ ), even when the region of the display containing the target is preceded by a spatial cue. The second panel shows that there was a secondary peak at Bin 3 (200-299 msec) for all conditions in Epochs 2 and 3, but importantly, cuing had very little influence on the relative number of early-phase responses. Finally, the third panel shows the data from the epochs that followed the cued epoch (i.e., Epochs 2, 3, and 4 following cued Epochs 1, 2, and 3, respectively), indicating that the relative RT distributions under these conditions were very similar to those for both cued and uncued epochs after the first look (Epoch 1).

These observations were tested by statistically comparing the proportions of early-phase (RT $<500 \mathrm{msec}$ ) and later-phase (RT $>500 \mathrm{msec})$ responses for each of the observers in each of the eight distributions shown in Figure 3. We refer to this measure as proportion $R R$. We readily admit that $500 \mathrm{msec}$ represents somewhat of an arbitrary cutoff, but we are using it merely as a matter of convenience for distinguishing between early- and laterphase responses. Readers should note that all the conclusions in this study remain unaltered if we adopt either a stricter (e.g., 400-msec cutoff) or a more lax criterion. Proportion RR was remarkably stable at .30 to .45 in all conditions shown in Figure 3, with the exception of the three cuing conditions in Epoch 1, where it was less than .04. An ANOVA revealed a significant interaction of epoch (1, average of 2 and 3 , epoch following cue) $\times$ cue (no cue, valid cue, invalid cue) overall $[F(1,112)=8.49$,

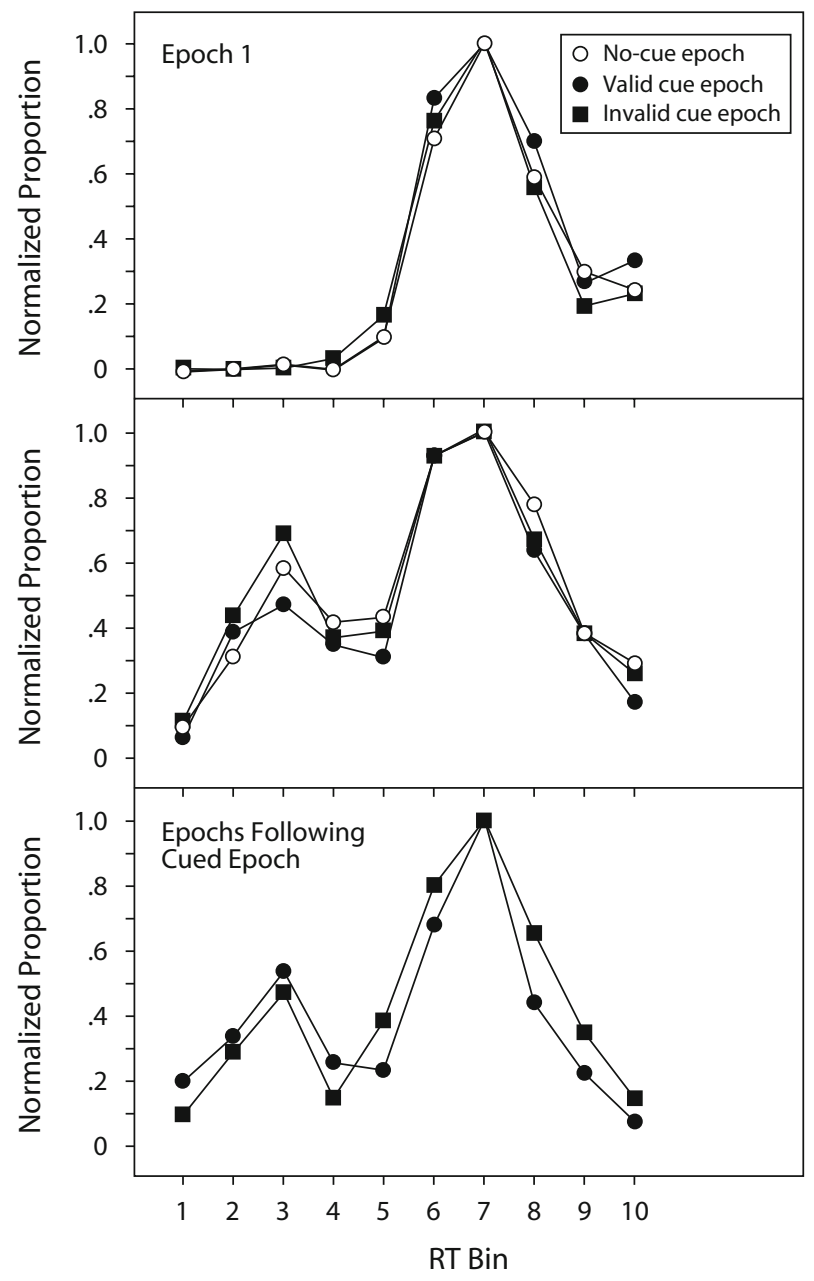

Figure 3. The normalized proportion of correct responses across all 10 bins (Bin $1=0-99 \mathrm{msec}$, Bin $2=100-199 \mathrm{msec}$, etc.) in an epoch in Experiment 1. For each cue condition, the bin with the highest frequency of responses was set to 1.0, in order to compare the relative distribution of responses in the other 9 bins. The middle panel shows average data from Epochs 2 and 3 of the no-cue condition and compares it with the average data of epochs that occurred immediately following a cue: cued Epoch 2 and cued Epoch 3. The bottom panel presents average data from the epochs following a cued epoch (Epoch 2 in the cued Epoch 1 condition, Epoch 3 in the cued Epoch 2 condition, and Epoch 4 in the cued Epoch 3 condition).

$\left.p<.01, M S_{\mathrm{e}}=.03\right]$, but when Epoch 1 was excluded, there were no longer any significant differences between conditions $(F<1.0)$.

\section{Discussion}

The results of Experiment 1 showed that a nonpredictive spatial cue, intended to draw attention involuntarily to one quadrant in advance of the search display, had a significant effect on the time required to identify the target. The cue decreased mean RT by about $200 \mathrm{msec}$ when it correctly indicated the target quadrant (valid), in comparison with when it indicated a nontarget quadrant (invalid). The same cue, however, had no effect on the shape of the response distribution following a look at the display. The 
proportion of correct responses that occurred in the first $500 \mathrm{msec}$ following a look (rapid resumption responses) was not increased by the cue, and the RT distributions, which were normalized to control for different baseline frequency of responses (Figure 3), also gave no hint that a valid cue altered either the portion of the distribution that peaked at Bin 3 (rapid resumption responses) or the portion of the distribution that peaked at Bin 7 (normal search responses). This result implies that the effect of the cue on mean RT was to increase the probability that the target was found in an earlier epoch when it was valid than when it was invalid. This result is analogous to a previous finding that the number of items in a search display had no effect on rapid resumption (Lleras et al., 2005): Proportion RR was not reduced when displays contained fewer items, although smaller set sizes did decrease overall RT by increasing the probability that the target would be found in an earlier epoch.

\section{EXPERIMENT 2}

\section{Predictive Spatial Cuing of the Target Region}

Although Experiment 1 revealed no relationship between involuntary (exogenous) spatial orienting and rapid resumption, it is possible that rapid resumption is associated with voluntary (endogenous) orienting to a region of the scene prior to a look. To test for this possibility, the design of Experiment 2 was identical to that of the previous experiment, with the exception that the cue, when it was presented, was $100 \%$ predictive of the quadrant of the search display containing the target. Observers were informed of the predictive nature of the cue and were told to use it to shorten their search.

\section{Method}

The method of Experiment 2 was identical to that of Experiment 1 , except that there were no invalid cued trials: Cues always perfectly predicted the target quadrant. The four experimental conditions were equally likely: On $25 \%$ of trials, there was never a cue (no cue), and on the remaining $75 \%$, a cue appeared indicating a target quadrant immediately before the first, second, or third look at the display ( $25 \%$ of trials in each condition). Eighteen students from the University of Illinois at Urbana-Champaign took part in this experiment in exchange for course credit. All were naive as to the purpose of the experiment and had normal or corrected-tonormal visual acuity.

\section{Results}

Figure 4 shows the frequency of correct responses for the first six epochs in the four cuing conditions (no cue, cued Epochs 1-3). Only a few responses (not shown) were longer than $6 \mathrm{sec}(<0.5 \%)$, and the accuracy of responses exceeded $96 \%$ overall. The RT distributions for no-cue trials (top panel in Figure 4) showed the typical pattern of rapid resumption. Following the first look at the search display (Epoch 1), fewer than 2\% of all responses occurred in the first 500 -msec period (4/226). In contrast, the first 500-msec period in each subsequent epoch contained more than $36 \%$ of the responses within an epoch $($ Epoch $2=215 / 633$, Epoch $3=158 / 493$, Epoch $4=$ $155 / 420$, Epoch $5=112 / 263$, Epoch $6=51 / 95$ ).
We examined the general effectiveness of the cue by computing mean correct RT in the four conditions for each observer. ANOVA indicated significant differences, with correct no-cue RT ( $M=2,695 \mathrm{msec})$ significantly greater than that in the cued conditions as a whole $(M=$ $1,723 \mathrm{msec})\left[F(1,17)=301.11, M S_{\mathrm{e}}=28,252, p<.01\right]$, and Look 1 cued RT $(M=951 \mathrm{msec})$ was significantly less than Look 2 cued RT $(M=1,813 \mathrm{msec})[F(1,17)=$ $1,101.02, p<.01]$, which, in turn, was less than Look 3 cued RT $(M=2,316 \mathrm{msec})\left[F(1,17)=374.49, M S_{\mathrm{e}}=\right.$ $17,653, p<.01]$. These analyses confirmed that predictive cuing of the target quadrant had a large effect on search time overall.

Figure 5 shows the relative distribution of correct responses within an epoch for the various cuing conditions. The first panel (Epoch 1) shows that, although spatial cuing had the systematic effect of increasing the relative frequency of responses in Bins 5-7 (400-700 msec), there were nonetheless very few early-phase responses $(\mathrm{RT}<500 \mathrm{msec})$. The middle panel is the most striking in showing a pattern of dissociation between spatial cuing and the rapid resumption phenomenon. Here spatial cuing increases responses over no cue in the range of 500-700 msec. However, at the same time, the early-phase responses normally associated with a second or third look at a display, evident in the no-cue condition, are virtually absent in the cued condition and no longer represent a significant mode of responses. This implies that, whereas a predictive spatial cue is able to increase the overall speed of response, as well as the likelihood of a response in the 400-1,000 msec range, it does not contribute to a greater proportion of rapid resumption responses. In fact, it did the opposite, actually reducing the relative frequency of early-phase responses in favor of later-phase responses. The third panel shows the data from Epochs 2, 3, and 4 following a cue (i.e., following cues in Epochs 1, 2, and 3, respectively). The results show that, following the second look at a spatially cued target region, a vast majority of responses were in the rapid resumption period $(\mathrm{RT}<$ $500 \mathrm{msec}$ ).

These observations were tested by statistically comparing the proportions of early-phase (RT $<500 \mathrm{msec}$ ) and later-phase (RT $>500 \mathrm{msec}$ ) responses for each of the observers in each of the five distributions shown in Figure 5. ANOVA indicated a significant interaction of epoch (1, average of 2 and 3 , epoch following cue) $\times$ cue (no cue, cued) $\left[F(2,34)=23.31, M S_{\mathrm{e}}=.005, p<.01\right]$. Proportion $\mathrm{RR}$ in Epoch 1 was less than .01 for the uncued condition and .09 in the cued condition, a significant difference $[F(1,17)=4.94, p<.03]$ due entirely to the small increase of responses in Bin 4. In Epoch 2, the direction of these differences was reversed, with the proportion RR averaging . 35 in the no-cue condition and only .23 in the cued condition $[F(1,17)=10.85, p<.01]$. Finally, proportion $\mathrm{RR}$ in the look immediately following the cued epoch was more than .65 , which was significantly greater than that for no cue in Epochs 2 and $3[F(1,17)=147.15, p<.01]$ and greater than that following any cued epochs $[F(1,17)=$ $247.71, p<.01]$. There were no differences among the second look after cue epochs $\left(F<1.0, M S_{\mathrm{e}}=.015\right)$. 

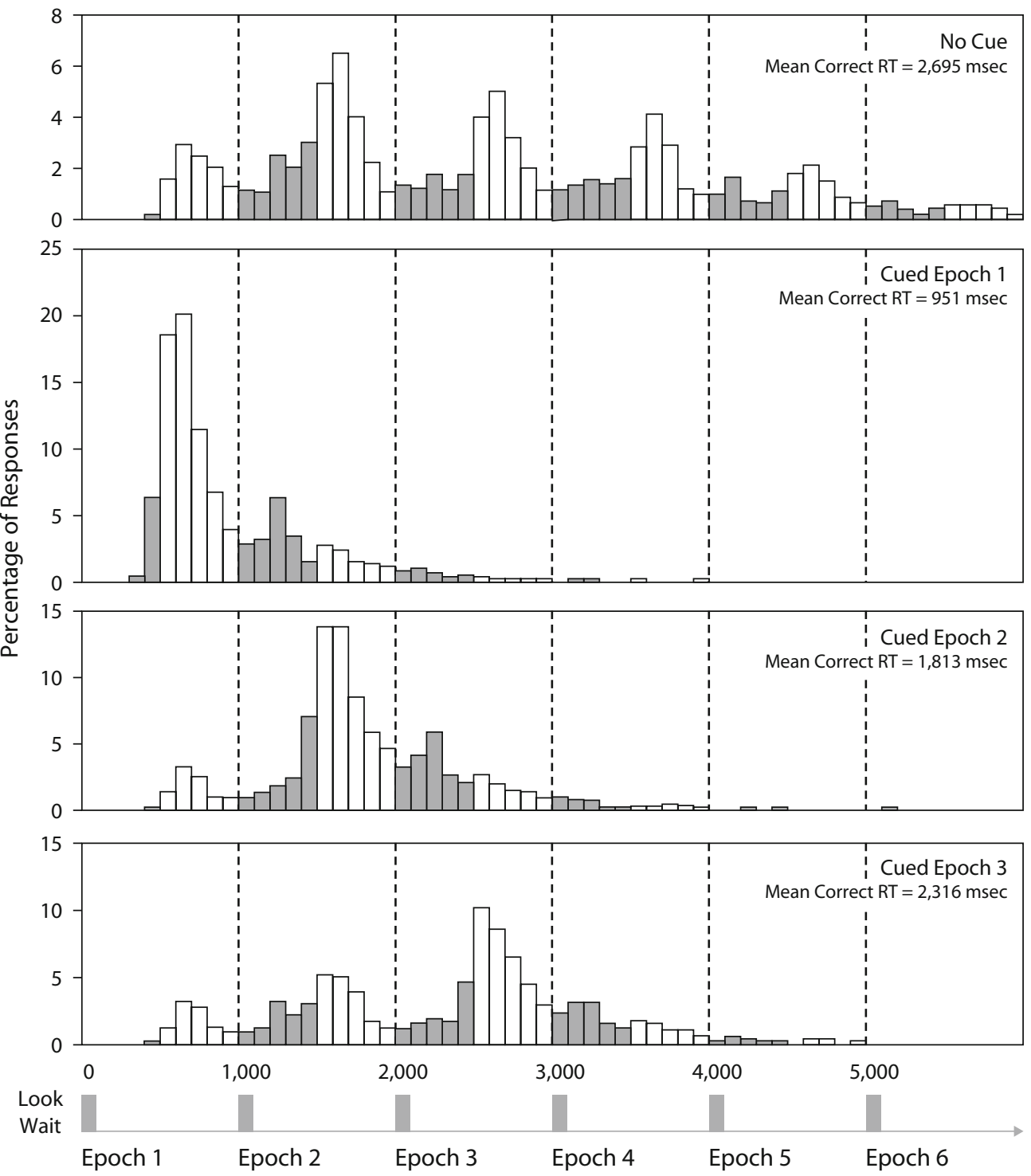

Figure 4. Frequency distributions of correct response times (RTs) in Experiment 2 as a function of cue condition. Histograms shaded in gray are responses that occured within 500 msec of a look, the operational definition of rapid resumption.

\section{Discussion}

Experiment 2 showed that a spatial cue that correctly predicted the target region in a search display led to a significant improvement in search times. This was evident in the significant decrease in mean RT on cued trials, and it was evident in the normalized response distributions, which showed that the leading edge of the cued distribution began about $100 \mathrm{msec}$ in advance of the distribution corresponding to a first look at a display. Yet this shift toward earlier responses with a predictive cue still occurred primarily within the normal search period (500-999 msec).

At the same time, the predictive cue had no positive effects on the rapid resumption effect. In fact, when the target was cued in advance of epochs that otherwise showed rapid resumption (second and subsequent looks at the display), the predictive cue significantly reduced the proportion of responses that fell within the rapid resump- tion period (0-499 msec). None of the cued distributions showed any evidence of a secondary peak at Bin 3 (300$399 \mathrm{msec}$ ). The cue did have a positive impact on rapid resumption on the second look that occurred following the cue. In these epochs, proportion RR rose above .65, which is significantly higher than the proportion RR observed without a cue (about .35 in this experiment).

\section{EXPERIMENT 3}

\section{Predictive Spatial Cuing of the Target Location}

Focusing spatial attention on one quadrant of a search display prior to the appearance of the items effectively reduced the number of items that had to be inspected during a search. Yet spatial orienting of this kind had no influence on the proportion RR, either when it was involuntary (Experiment 1) or when it was voluntary (Experiment 2). As a 


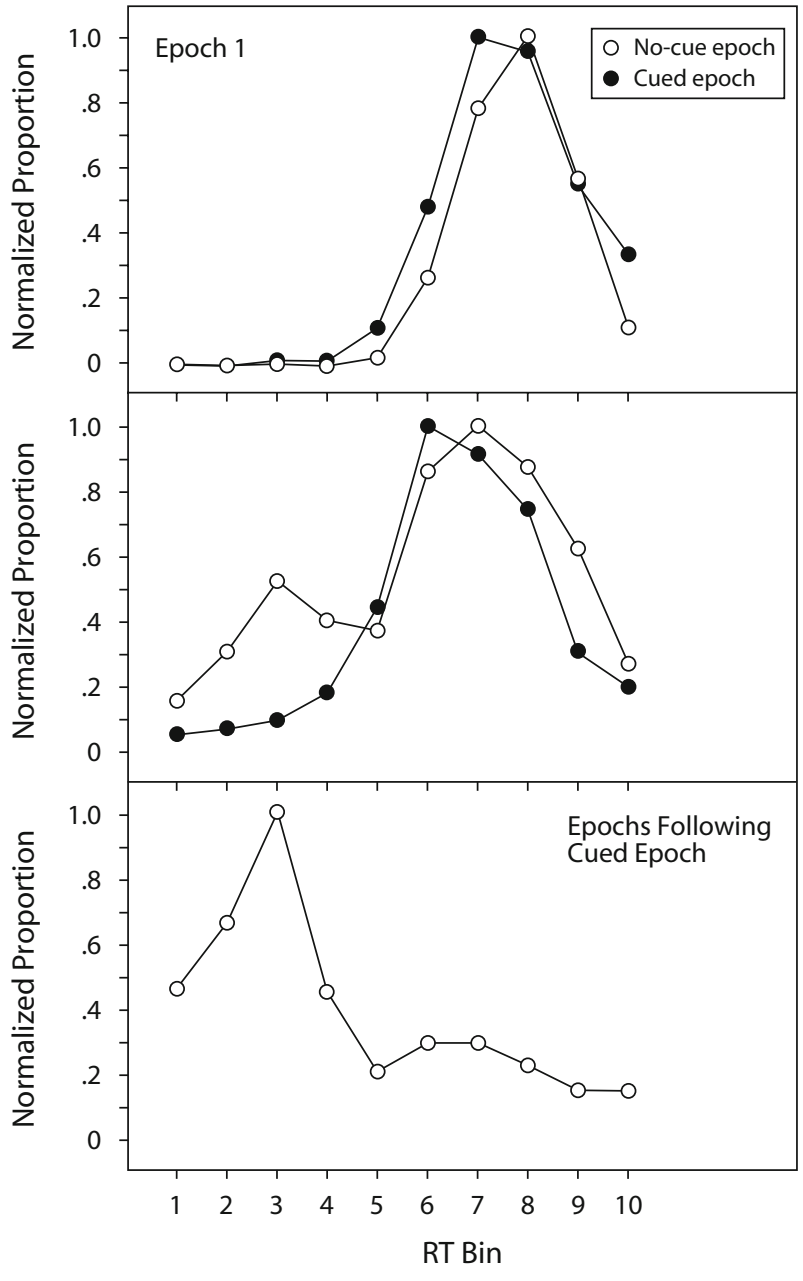

Figure 5. The normalized proportion of correct responses across all 10 bins $(B i n 1=0-99 \mathrm{msec}$, Bin $2=100-199 \mathrm{msec}$, etc.) in an epoch in Experiment 2. For each cue condition, the bin with the highest frequency of responses was set to 1.0 , in order to compare the relative distribution of responses in the other 9 bins. The middle panel shows average data from Epochs 2 and 3 of the no-cue condition and compares it with the average data of epochs that occurred immediately following a cue: cued Epoch 2 and cued Epoch 3. The bottom panel presents average data from the epochs following a cued epoch (Epoch 2 in the cued Epoch 1 condition, Epoch 3 in the cued Epoch 2 condition, and Epoch 4 in the cued Epoch 3 condition).

final stringent check on the possible relationship between spatial orienting and rapid resumption, the present experiment examined voluntary spatial orienting to the specific location of the target item in advance of the search display. The methods and design of Experiment 3 were identical to those of Experiment 2, with the exception that the cue was now $100 \%$ predictive of the location of the target within the search display.

\section{Method}

Unlike in Experiments 1 and 2, the cuing dots were presented within the search grids, at the outermost corners of the grid cell that would eventually contain the target in the subsequent search display, so that the dots would surround but not overlap with the target item.
Four conditions analogous to those in Experiment 2 were examined: no cue, Look 1 cued, Look 2 cued, and Look 3 cued. All conditions were equally likely. Twenty students from the University of Illinois at Urbana-Champaign took part in this experiment in exchange for course credit. All were naive as to the purpose of the experiment and had normal or corrected-to-normal visual acuity.

\section{Results}

Figure 6 shows the frequency of correct responses for the first six epochs. Only a few responses were not shown, because they were longer than $6 \sec (<5.0 \%)$; the accuracy of responses exceeded $95 \%$ overall. The RT distribution for no-cue trials (Figure 6, top panel) showed the typical pattern of rapid resumption. Following the first look at the search display (Epoch 1), fewer than $1.5 \%$ of all responses occurred in the first 500-msec period (8/564). In contrast, the first 500 -msec period following each subsequent look contained more than $44 \%$ of the responses within an epoch (Look $2=412 / 856$, Look $3=264 / 608$, Look $4=$ 169/410, Look $5=92 / 235$, Look $6=63 / 137$ ).

We examined the general effectiveness of the cue by computing mean correct RT in the four conditions for each observer. ANOVA indicated significant differences, with no-cue RT $(M=2,561 \mathrm{msec})$ being significantly greater than RT for the cued conditions as a whole $(M=$ $1,308 \mathrm{msec})\left[F(1,19)=178.60, M S_{\mathrm{e}}=87,915, p<.01\right]$. Cued Epoch 1 RT $(M=538 \mathrm{msec})$ was significantly lower than that for cued Epoch $2(M=1,430 \mathrm{msec})[F(1,19)=$ 931.06, $p<.01$ ], which in turn was lower than that for cued Epoch $3(M=1,949 \mathrm{msec})[F(1,19)=242.60$, $\left.M S_{\mathrm{e}}=11,082, p<.01\right]$. These analyses confirmed that cuing the target location provided a large benefit on search time overall.

Figure 7 shows the relative distribution of correct responses within an epoch for the various cuing conditions. The first panel (Epoch 1), as in the previous experiment, showed that spatial cuing increased the relative frequency of responses in Bins 5 and 6 (400-599 msec). The peak of the response distribution in Epoch 1 was 100 msec earlier with a target-specific cue than with no cue. However, the middle panel shows that the distribution of responses associated with the cue in Epochs 2 and 3, which peaked in Bin 5 (400-499 msec), was still not as early as that associated with a second look in the absence of any cue. The first peak in the no-cue distribution was centered on Bin 3 (200-299 msec). Finally, the third panel (epoch following cued epoch) showed a two-peaked distribution of responses similar to those in the absence of a cue.

These observations were tested by statistically comparing the proportion of early-phase (RT $<500 \mathrm{msec})$ and later-phase (RT $>500 \mathrm{msec}$ ) responses for each of the observers in each of the five distributions shown in Figure 7. ANOVA indicated a significant interaction of epoch (1, average of 2 and 3 , epoch following cue) $\times$ cue (no cue, cued) $\left[F(4,76)=56.16, M S_{\mathrm{e}}=.025, p<.01\right]$. Proportion RR in Epoch 1 was .01 for the uncued condition and .36 in the cued condition, a significant difference $[F(1,19)=56.81, p<.03]$ due entirely to the increases in responses in Bin 5 (400-499 msec). In Epoch 2, the direction of these differences was reversed, with proportion RR 

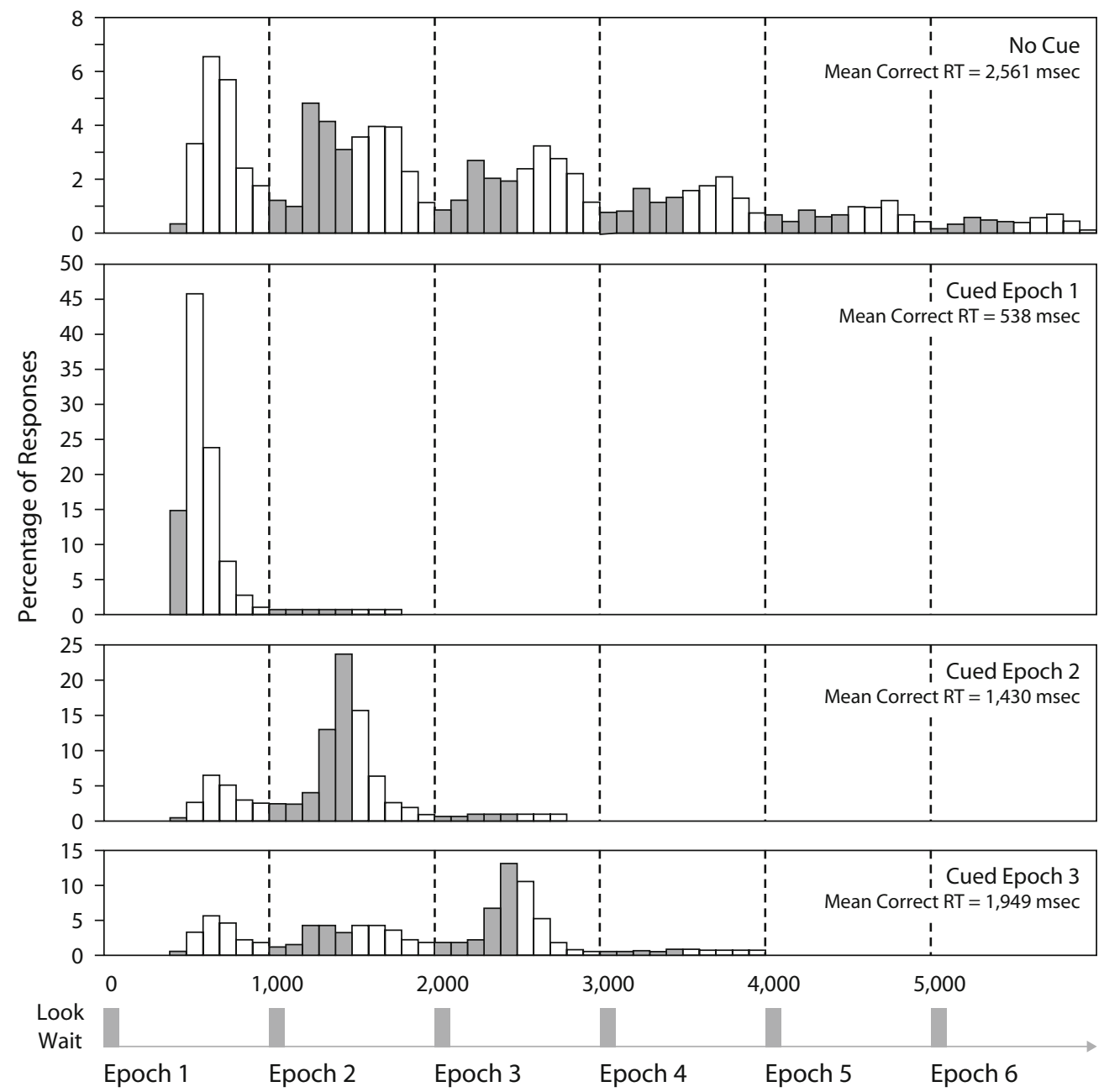

Figure 6. Frequency distributions of correct response times (RTs) in Experiment 3 as a function of cue condition. Histograms shaded in gray are responses made within $500 \mathrm{msec}$ of a look, the operational definition of rapid resumption.

averaging .53 in the no-cue condition and only .38 in the cued condition $[F(1,19)=8.20, p<.01]$. Finally, proportion RR in the look immediately following the cued epoch was more than .49 , which was similar to that for no cue in Epochs 2 and $3[F(1,19)<1.0]$ and greater than that following the cued epochs $[F(1,19)=6.16, p<.02]$.

Response speed and accuracy. An important question relevant to the interpretation of all three experiments here and to the data from previously published experiments on rapid resumption is how the phenomenon of rapid resumption is related to the response biases of the observer. For example, is it possible that these early-phase responses reflect a tendency for observers to be overly cautious, taking the opportunity to have one more look, even though it is unnecessary, before making their response? If so, a more liberal response criterion should reduce and may even eliminate the RR phenomenon.

We addressed this question in the present data in two ways. First, we examined the relationship between response accuracy (proportion of errors) and proportion RR in the three experiments. The accuracy measures we used included the proportion of errors made on all no-cue trials (uncontaminated by any effects of spatial cuing) and the percentage of errors made overall (across both cued and uncued trials). The speed of response measures included the proportion of RR responses (RT $<500 \mathrm{msec}$ ) in Epochs 2 and beyond in the no-cue condition (uncontaminated by any effects of spatial cuing) and the proportion of RR responses made overall (across all trial types).

This yielded data for a total of 53 participants $(n \mathrm{~s}=15$, 18 , and 20 in the three experiments), with average error rates ranging from .01 to .16 in the no-cue condition and from .01 to .14 overall. Proportion RR ranged from .15 to .68 in the no-cue condition and from .17 to .72 overall. The correlation between the two error measures was very strong $(r=.72)$, as was the correlation between the two measures of rapid resumption $(r=.81)$. Yet the correlations between the error measures and the measures of rapid resumption were all near $0(r=-.03$ to +.22$)$. The slight positive correlations suggest that, if anything, higher error rates tend to be associated with a larger proportion of rapid resumption responses. This is opposite 


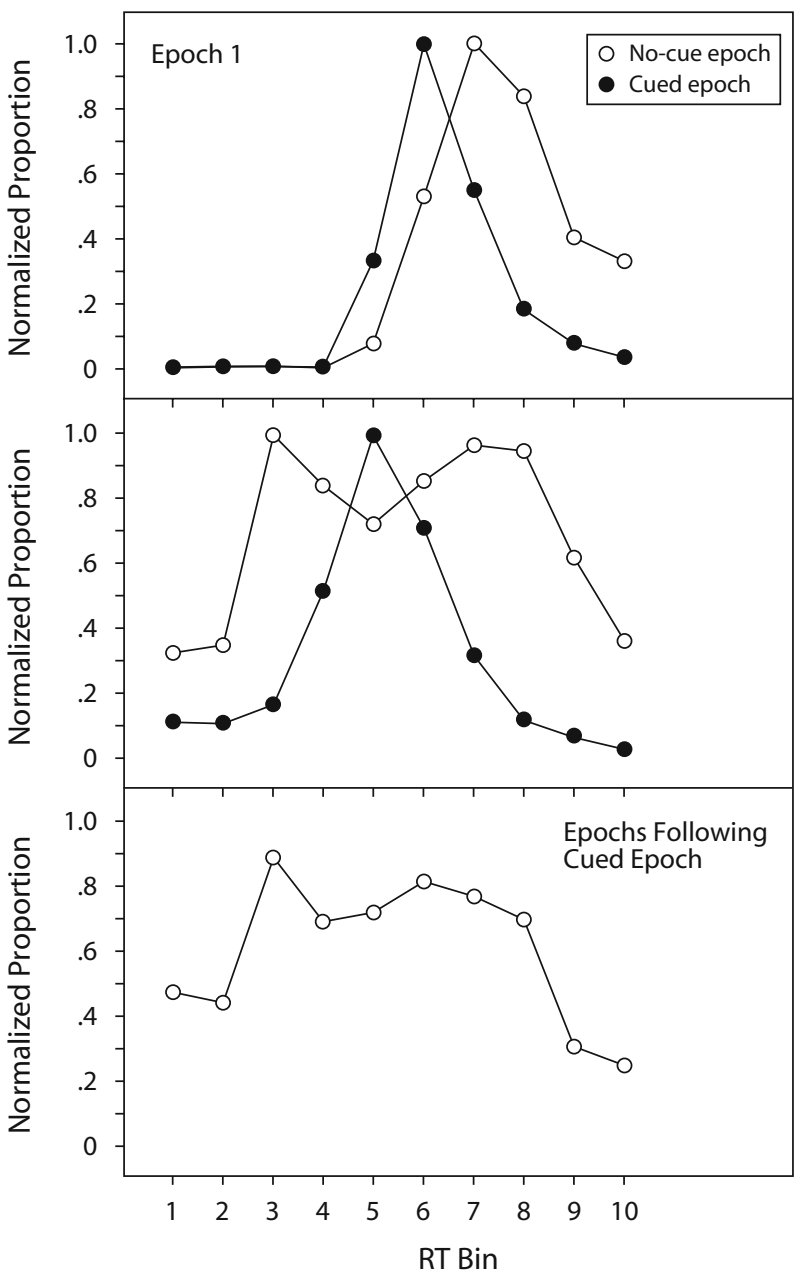

Figure 7. The normalized proportion of correct responses across all 10 bins $(B i n 1=0-99 \mathrm{msec}$, Bin $2=100-199 \mathrm{msec}$, etc.) in an epoch in Experiment 3. For each cue condition, the bin with the highest frequency of responses was set to 1.0 , in order to compare the relative distribution of responses in the other 9 bins. The middle panel shows average data from Epochs 2 and 3 of the no-cue condition and compares it with the average data of epochs that occurred immediately following a cue: cued Epoch 2 and cued Epoch 3. The bottom panel presents average data from the epochs following a cued epoch (Epoch 2 in the cued Epoch 1 condition, Epoch 3 in the cued Epoch 2 condition, and Epoch 4 in the cued Epoch 3 condition).

to the hypothesis that a liberal response criterion might reduce or eliminate the rapid resumption phenomenon.

Our second approach to the question of whether response biases contribute to early-phase responses involved inspection of the distribution of response errors over time. When we combined data from all three experiments, in order to maximize our opportunity for detecting a pattern in these relatively rare responses (53 observers yielded a total of 1,356 errors), we observed that errors were distributed over time in a way that was very similar to that for correct responses. In Epoch 1, almost no responses were made in less than $500 \mathrm{msec}(<5 \%)$, whereas in Epochs 2-6, errors were distributed between the early and late phases of each epoch in much the same way as were correct responses $(493 / 977=.51$ were made in $<500 \mathrm{msec} ; 484 / 977=.49$ were made $>500 \mathrm{msec}$ ). However, when we examined the proportion of errors made in the early phase separately for no-cue and cued epochs, it was clear that the cue contributed to an increase in early-phase errors $(41.8 \%$ of errors $<500 \mathrm{msec}$ in the no-cue condition; $55.4 \%$ of errors $<500 \mathrm{msec}$ in the cued conditions; $\left.\chi^{2}=15.74, p<.001\right)$. Taken together, these analyses do not support the hypothesis that a liberal response bias contributes to early-phase correct responses. However, what the analyses do show is that the spatial cue used in this experiment contributed to early-phase response errors over and above the baseline likelihood of making a response error during this period.

\section{Discussion}

Experiment 3 showed that a spatial cue in the precise target location increased search speed to an even greater extent than did a cue that was correctly predictive of only the general target region (previous experiment). The distribution of normal search responses was shifted forward by more than $100 \mathrm{msec}$ by this cue. The target location cue also guaranteed search success in the epoch following the cued epoch to a greater extent than did the general region cue, because very few responses occurred more than one epoch after the cue had been presented. Yet this large increase in responses that were facilitated by the target cue occurred primarily within the normal search period (500-999 msec following a look). The proportion of early-phase responses ( $0-499 \mathrm{msec})$ was not increased by the presence of this otherwise highly effective search cue, with the exception of Bins 4 and 5 (300-499 msec). In fact, the proportion RR in the target cue condition was still significantly less than the proportion RR following the second and third looks in the no-cue condition (in particular, see Bin 3 in the second panel of Figure 7). It was also notable that there was no hint of an early-phase peak of responses in the cued condition, both when the target in the first look was cued (top panel) and when the second and third looks were cued (second panel). From this, we conclude that focused spatial attention does not in itself contribute to the phenomenon of rapid resumption of an interrupted search. Moreover, cuing the location of the target in advance serves to actively reduce the likelihood of an early-phase correct response at the same time that it vastly increases the likelihood of a correct late-phase response.

\section{GENERAL DISCUSSION}

These three experiments were conducted to determine whether focused spatial attention plays a causal role in the rapid resumption of an interrupted search. Specifically, we asked the question, Do the fast correct RTs that are observed when an interrupted visual search is resumed (Lleras et al., 2005) occur because of a coincidence between the spatial focus of the observer's attention and the location of the target in the display? This is important to consider, because it has long been known that focused spatial attention provides a number of benefits to percep- 
tion, including faster and more accurate responding (Luck \& Vecera, 2002; Yeshurun \& Carrasco, 1999), immunity from backward masking (Enns, 2004), and success in detecting changes to scenes (O'Regan et al., 2000; Rensink, 2000). If spatial orienting also contributes to the rapid resumption of an interrupted search, this phenomenon should properly be reinterpreted as an instance of "lucky" spatial orienting, rather than as evidence of an implicit perceptual hypothesis-testing process that is awaiting confirmation, as proposed by Lleras et al. $(2005,2007)$.

Experiment 1 showed that exogenously cuing observers in advance to the general region in which the target later appeared produced a significant reduction in average search time. This is the traditional spatial cuing effect many others have noted previously in visual search experiments (Cheal, Lyon, \& Gottlob, 1994). It is also evident in Figure 2 when valid cue (second and fourth panels) and invalid cue (third and fifth panels) conditions are compared. This comparison shows an increased number of correct responses for valid cues in the latter half of each epoch (i.e., RT of 500-1,000 msec). Yet, despite search benefiting in this way, observers in Experiment 1 were unable to use this spatial cue to produce more early-phase responses to a target immediately following the presentation of the cue. This is shown by the near absence of any rapid resumption responses (RT $<500 \mathrm{msec}$ ) when a cue preceded the first look at the display (e.g., valid cue Epoch 1). It is also shown in Figure 3 as the relative proportion of rapid resumption responses in Epochs 2 and 3 following a valid cue (middle panel). These data show rapid resumption at no greater rate following a valid cue than following an invalid cue or even no cue, in the second epoch and beyond. The epoch that followed on the heels of a cued epoch (lower panel in Figure 3) also showed no influence of the cue on rapid resumption responses that normally occur in the second and subsequent looks at a search display.

When we explored the role of a perfectly predictive (endogenous) region cue in Experiment 2, the results were not much different. The first panel of Figure 5 shows that the relative frequency of early-phase responses in the first epoch did not differ much, depending on whether there was a cue that reduced the effective number of search items to a quarter of the total. Having completely reliable evidence of the region in which the target would appear did, however, have a large benefit on search success, in that many more correct responses were made in the second half of the epoch (RT from 500 to $1,000 \mathrm{msec}$ ). However, this foreknowledge still did not allow observers to produce early-phase responses ( $\mathrm{RT}<500 \mathrm{msec}$ ) at a greater rate.

When we directed observers' attention to the exact location of the target in Experiment 3, the cue was even more overwhelmingly effective at ensuring search success in the epoch immediately following the presentation of the cue. Figure 6 (second panel) shows that a vast majority of the correct responses occurred within $1,000 \mathrm{msec}$ following the first look. Yet this highly effective spatial cue nonetheless still failed to produce the many early-phase responses that characterize rapid resumption. In summary, these three experiments reveal dissociable effects of focused spatial attention and the rapid resumption of search. Although spatial cuing can increase the speed and accuracy of the perceptual processes of visual search, it does not in itself contribute to the rapid resumption phenomenon. We believe that rapid resumption occurs, as we have described elsewhere (Enns \& Lleras, 2008; Lleras et al., 2005,2007 ), because observers are taking advantage of the perceptual processing that begins following one look (activation and prediction) and are using it to complete the perception cycle (confirmation) in a second look.

In two previous reports, Lleras et al. $(2005,2007)$ examined the conditions that give rise to rapid resumption. Their experiments were guided by the theoretical idea that rapid resumption is an index of the benefit of having already created a perceptual hypothesis about the target (on the basis of the first look), and prior to the appearance of the second look, that need only be confirmed in the second look for conscious perception of the target to occur. To illustrate this idea in greater detail, we will refer to the last look at the display, prior to making a response on any given trial, as Look $L$. The authors proposed that, on trials on which rapid resumption was observed, observers had generated a perceptual hypothesis about the target on the previous look (i.e., on Look L-1). But because sensory information regarding the target was removed from the visual system before this hypothesis could be confirmed, observers did not become aware of the target. In other words, the perceptual hypothesis about the target remained unconfirmed. However, upon re-presentation of the display (i.e., on Look L), the partial information about the target contained in this perceptual hypothesis was combined with current sensory information to accelerate target processing, resulting in both faster awareness of the target and faster responses.

Within this framework, the present results can be easily accounted for: Cuing attention to the target location cannot produce rapid resumption results, because, regardless of how effective the cue is, it is no substitute for the formation of a perceptual hypothesis about the target's identity. Whereas focused attention may hasten the perceptual processing at the cued location, processing of the target features necessary to confirm its identity must start from scratch. In contrast, on rapid resumption trials, the perceptual hypothesis created on Look L-1 allows for much more efficient processing of (and then responding to) the target simply because some of the critical perceptual processing has already taken place before that look occurred. Note, too, that the generation of a perceptual hypothesis can induce a motor preparation for the response that is strongly associated with the features in that perceptual hypothesis, even before observers become aware (or sometimes even in the absence of awareness) of the target (e.g., Lleras \& Enns, 2004, 2006; Lleras et al., 2007). It is therefore likely that this motor preparation is also partly responsible for the extremely fast responses observed in rapid resumption.

This framework can also make sense of the pattern observed in the bottom panel of Figure 5: On the epoch following the cued epoch (Epoch $\mathrm{L}+1$ ), there is a dramatic 
increase in rapid resumption responses. This observation is consistent with the cue's having increased the likelihood that a perceptual hypothesis about the target will be created on Look L (immediately after the cue). If the hypothesis is confirmed on that epoch, a rise of normal responses is observed (middle panel). If, on the other hand, the hypothesis does not have a chance to be confirmed on Look L, it will certainly be ready for confirmation on Look $\mathrm{L}+1$. This explains why a vast majority of responses on Epoch $\mathrm{L}+1$ are of the rapid resumption kind.

The difference between the bottom panels in Figures 5 and 7, then, is also quite informative: When the cue is spatially vague (Figure 5; the cue only generally indicates the quadrant containing the target), almost all responses on Epoch $\mathrm{L}+1$ are rapid resumption responses, whereas when the cue is spatially accurate (Figure 7; the cue perfectly directs attention to the target location), the increase in rapid resumption responses is more subdued. This follows, because with spatially accurate cues, the likelihood of forming and confirming a hypothesis about the target during a single epoch is substantial (as is indicated by differences in overall response frequencies between cued epochs in Figures 4 and 6). In sum, the present results suggest that a key ingredient for rapid resumption responses is spatial proximity to the target, coupled with spatial uncertainty about it (as was the case in Experiment 2): Under these conditions, the chances that perceptual hypotheses about the target will be created on one look and confirmed on a subsequent look seem maximal.

This tentative conclusion fits well with a recent report in which eye movements were recorded during an interrupted visual search task (van Zoest et al., 2007). The primary factor determining whether any given look would produce a rapid resumption response (within $500 \mathrm{msec}$ of last display onset) or a later response (from 500 to $1,000 \mathrm{msec}$ after the last display onset) was not the distance between current eye position and the target on Look L. In fact, eyetarget distance on Look L did not discriminate between the two types of responses. However, the eye-target distance on Look $\mathrm{L}-1$ did predict the relative frequency of rapid resumption responses, with increased rapid resumption correlated with decreased eye-target distance. This is consistent with the present data in supporting the idea that what is crucial in rapid resumption is to have the opportunity on a previous look to do some (albeit incomplete) processing of the target.

In van Zoest et al. (2007), this proposal was partially confirmed by using fixation-contingent search displays in two experiments. In these experiments, the target was presented to the center of gaze, regardless of where the observer fixated. This meant that, after one of the waits between looks, the search display reappeared and the target was exactly at the center of the observer's gaze, regardless of where they were looking. Just as in the present study with spatial cues, giving the observer prior information about target location sharply increased the likelihood of a successful search within the next two epochs. In fact, search success in the look immediately following gaze-contingent target relocation was $75 \%$ (van Zoest et al., 2007, Experiment 2), and in the look immediately following a spatial cue that was completely predictive of the target location it was over $80 \%$ (the present Experiment 3). Yet this high baseline rate of search success did not contribute to a higher proportion of rapid resumption responses in either case. Those responses still occurred primarily after the second look at a gaze-contingent target or following a predictive cue.

One of the limitations of the fixation-contingent studies (van Zoest et al., 2007) is that the (fixation-contingent) rearrangement of the target from look to look in those studies also involved altering the stability of the search display. Because the target no longer remained in a fixed location, it is possible that observers were somewhat slower to respond on those trials simply because the new information in each display did not match their expectations (either consciously or unconsciously) about what would be at a given location. Moreover, the rearrangement of the target also changed the spatial configuration of items at the fixated region, and previous studies have reported that changing the configuration of the search display in this way disturbs rapid resumption (Jungé, Brady, \& Chun, 2008; Lleras et al., 2005, 2007). In contrast to these limitations, the present spatial cuing manipulations did not alter the information in the search display; they simply allowed observers to form a spatial expectancy about where to look prior to the search display and during one of the waits between looks. As such, the present finding that spatial orienting is independent of the rapid resumption phenomenon provides converging evidence that rapid resumption responses are not the consequence of either "lucky" eye fixations (van Zoest et al., 2007) or "lucky" spatial expectancies. Instead, it suggests that rapid resumption is critically dependent on observers' having the opportunity to partially process the target (Lleras et al., 2007) and the spatial layout of items immediately around it (Jungé et al., 2008) during a prior look.

\section{AUTHOR NOTE}

This research was supported by research NSF Grant 0527361 and an NSERC Discovery Research Grant to J.T.E. Address correspondence to J. T. Enns, Department of Psychology, University of British Columbia, 2136 West Mall, Vancouver, BC, V6T 1Z4 Canada (e-mail: jenns@, psych.ubc.ca)

\section{REFERENCES}

Brainard, D. H. (1997). The Psychophysics Toolbox. Spatial Vision, 10, 433-436. doi:10.1163/156856897X00357

Cheal, M. L., Lyon, D. R., \& Gottlob, L. R. (1994). A framework for understanding the allocation of attention in location-precued discrimination. Quarterly Journal of Experimental Psychology, 47A, 699-739.

Di Lollo, V., EnNs, J. T., \& Rensink, R. A. (2000). Competition for consciousness among visual events: The psychophysics of reentrant visual processes. Journal of Experimental Psychology: General, 129, 481-507. doi:10.1037/0096-3445.129.4.481

ENNS, J. T. (2004). Object substitution and its relation to other forms of visual masking. Vision Research, 44, 1321-1331.

EnNs, J. T., \& Lleras, A. (2008). What's next? New evidence for prediction in human vision. Trends in Cognitive Sciences, 12, 327-333. doi:10.1016/j.tics.2008.06.001

Henderson, J. M., \& Hollingworth, A. (2003). Eye movements, vi- 
sual memory, and scene representation. In M. A. Peterson \& G. Rhodes (Eds.), Perception of faces, objects, and scenes: Analytic and holistic processes (pp. 356-383). New York: Oxford University Press.

Jungé, J. A., Brady, T. F., \& Chun, M. M. (2008). The contents of perceptual hypotheses: Evidence from rapid resumption of interrupted visual search. Manuscript submitted for publication.

LLERAS, A., \& ENNS, J. T. (2004). Negative compatibility or object updating? A cautionary tale of mask-dependent priming. Journal of Experimental Psychology, 133, 475-493. doi:10.1037/0096-3445.133.4.475

Lleras, A., \& ENNS, J. T. (2006). How much like a target can a mask be? Geometric, spatial, and temporal similarity in priming - A reply to Schlaghecken and Eimer. Journal of Experimental Psychology: General, 135, 495-500. doi:10.1037/0096-3445.135.3.495

LleRAs, A., RENSINK, R. A., \& ENNS, J. T. (2004). Rapid resumption is modulated by high-level strategies. Journal of Vision, 4, 180

Lleras, A., Rensink, R. A., \& EnNs, J. T. (2005). Rapid resumption of an interrupted search: New insights on interactions of vision and memory. Psychological Science, 16, 684-688. doi:10.1111/j.1467 -9280.2005.01596.x

Lleras, A., Rensink, R. A., \& Enns, J. T. (2007). Consequences of display changes during interrupted visual search: Rapid resumption is target specific. Perception \& Psychophysics, 69, 980-993.

LucK, S. J., \& VeCERA, S. P. (2002). Attention. In H. Pashler \& S. Yantis (Eds.), Stevens' handbook of experimental psychology: Vol. 1. Sensation and perception (pp. 235-286). Hoboken, NJ: Wiley.
O’Regan, J. K., Deubel, H., Clark, J. J., \& Rensink, R. A. (2000). Picture changes during blinks: Looking without seeing and seeing without looking. Visual Cognition, 7, 191-211.

Pelli, D. G. (1997). The VideoToolbox software for visual psychophysics: Transforming numbers into movies. Spatial Vision, 10, 437-442.

ReNsink, R. A. (2000). Visual search for change: A probe into the nature of attentional processing. Visual Cognition, 7, 345-376. doi:10.1080/ 135062800394847

RIEBE, C., \& ENNS, J. T. (2008). What's special about looking for your own face? Rapid resumption yields insights on the visual search process. Manuscript in preparation.

SPERLING, G. (1960). The information available in brief visual presentations. Psychological Monographs, 74, 498.

van Zoest, W., Lleras, A., Kingstone, A. F., \& EnNs, J. T. (2007). In sight, out of mind: The role of eye movements in the rapid resumption of visual search. Perception \& Psychophysics, 69, 1204-1217.

YeshuRUn, Y., \& CARRASCO, M. (1999). Spatial attention improves performance in spatial resolution tasks. Vision Research, 39, 293-306. doi:10.1016/S0042-6989(98)0014-X

(Manuscript received January 23, 2008; revision accepted for publication October 6, 2008.) 